\title{
Evaluation of Essential Oils and Extracts of Rose Geranium and Rose Petals as Natural Preservatives in Terms of Toxicity, Antimicrobial, and Antiviral Activity
}

\author{
Chrysa Androutsopoulou ${ }^{1}$, Spyridoula D. Christopoulou ${ }^{2}$, Panagiotis Hahalis ${ }^{3}$, Chrysoula Kotsalou ${ }^{1}$, \\ Fotini N. Lamari ${ }^{2} \mathbb{D}$ and Apostolos Vantarakis $1, * \mathbb{D}$ \\ 1 Department of Public Health, Faculty of Medicine, University of Patras, 26504 Patras, Greece; \\ chrysandr@gmail.com (C.A.); chrysoulakotsalou@gmail.com (C.K.) \\ 2 Laboratory of Pharmacognosy \& Chemistry of Natural Products, Department of Pharmacy, University of Patras, \\ 26504 Patras, Greece; spiridoulaxr@windowslive.com (S.D.C.); flam@upatras.gr (F.N.L.) \\ 3 Tentoura Castro-G.P. Hahalis Distillery, 26225 Patras, Greece; hahalis@tentoura.gr \\ * Correspondence: avanta@upatras.gr
}

Citation: Androutsopoulou, C.; Christopoulou, S.D.; Hahalis, P.; Kotsalou, C.; Lamari, F.N.; Vantarakis, A. Evaluation of Essential Oils and Extracts of Rose Geranium and Rose Petals as Natural Preservatives in Terms of Toxicity, Antimicrobial, and Antiviral Activity. Pathogens 2021, 10, 494. https://doi.org/10.3390/ pathogens10040494

Academic Editor: Valentina

Virginia Ebani

Received: 24 March 2021

Accepted: 15 April 2021

Published: 19 April 2021

Publisher's Note: MDPI stays neutral with regard to jurisdictional claims in published maps and institutional affiliations.

Copyright: (c) 2021 by the authors. Licensee MDPI, Basel, Switzerland This article is an open access article distributed under the terms and conditions of the Creative Commons Attribution (CC BY) license (https:// creativecommons.org/licenses/by/ $4.0 /)$

\begin{abstract}
Essential oils (EOs) and extracts of rose geranium (Pelargonium graveolens) and petals of rose (Rosa damascena) have been fully characterized in terms of composition, safety, antimicrobial, and antiviral properties. They were analyzed against Escherichia coli, Salmonella enterica serovar Typhimurium, Staphylococcus aureus, Aspergillus niger, and Adenovirus 35. Their toxicity and life span were also determined. EO of $P$. graveolens (5\%) did not retain any antibacterial activity (whereas at $100 \%$ it was greatly effective against $E$. coli), had antifungal activity against $A$. niger, and significant antiviral activity. Rose geranium extract (dilutions 25-90\%) $(v / v)$ had antifungal and antibacterial activity, especially against $E$. coli, and dose-dependent antiviral activity. Rose petals EO (5\%) retains low inhibitory activity against $S$. aureus and $S$. Typhimurium growth (about $20-30 \%$ ), antifungal activity, and antiviral activity for medium to low virus concentrations. Rose petals extract had significant antibacterial activity at dilutions of 25-90\%, especially against E. coli and S. Typhimurium, antifungal, and the most potent antiviral activity. None of the EOs and extracts were toxic in dilutions of up to $5 \%$ and $90 \%$, respectively. Finally, all materials had a life span of more than eight weeks. These results support the aspect that rose petals and rose geranium EOs, and extracts, have beneficial antimicrobial and antiviral properties and they can be used as natural preservatives.
\end{abstract}

Keywords: aromatic plants; essential oils; extracts; antimicrobial; antiviral; acute toxicity; lifetime

\section{Introduction}

Medicinal Aromatic Plants (M.A.P.) are a major part of the natural flora and considered an important resource in various fields, for instance the pharmaceutical, cosmetic, food, fragrance, and perfumery industries [1]. Nowadays more than $80 \%$ of the world population relies on traditional herbal medicines to treat health issues [2,3]. More than 9000 plants have been identified and recorded for their therapeutic properties. About 1500 species are known for their aroma and taste [4]. Natural Aromatic Chemicals are in great demand in different fields such as cosmetics, food, pharmaceuticals, and perfumes. On the contrary, today's society is facing a negative opinion with the chemical preservatives. Therefore, organic foods are commonly produced without the use of chemicals, and their microbial load could be higher. Therefore, plant chemical compounds constitute an alternative to their maintenance $[5,6]$. EOs and extracts have been used for their aroma, taste, medicinal properties, and as bactericides and preservatives [7]. Therefore, EOs and extracts are a safe, environmentally friendly, cost-effective choice for nutrition and environmental protection. EOs and extracts have gained their role as preservatives due to certain chemical compounds they contain, such as terpenes, terpenoids, carotenoids, and phenolics [8,9]. Many EOs 
and extracts have anti-inflammatory, antibacterial, antifungal, antiviral, and antiseptic features [10-12].

Pelargonium graveolens (Thunb.) L'Hèr belongs to the Geraniaceae family and to the genus of Pelargonium, which includes more than 200 species. It is a perennial herbaceous species and has its roots in South Africa; however it is now growing in other places as an ornamental plant and is also cultivated for its use in the food and drink industry [13]. The main volatile compounds are oxygenated monoterpenes (64.3-74.2\%), but the essential oil composition greatly depends on the genotype, the environmental conditions, the agricultural practices of cultivation, and the time of collection [14].

Rosa damascena Herrm. belongs to the Rosaceae family and the genus of Rosa [15]. The major constituents in the EO are $\beta$-citronellol, geraniol, eugenol, 2-phenylethyl alcohol, methyleugenol, linalool, and aliphatic hydrocarbons [16]. The antimicrobial, antioxidant, analgesic, anti-inflammatory, antidiabetic, and antidepressant properties of $R$. damascena have been demonstrated in preclinical studies [17]. Its petals have attractive organoleptic properties and find numerous applications in cooking. They are also included in numerous food products and drinks.

The Food and Drug Administration (FDA) and the Environment Protection Agency (EPA) in the USA have recognized many essential oils as "safe products" for food and beverage consumption [18]. However, their adjustment as food preservatives demands detailed awareness about their properties and concentrations.

The purpose of this study was to fully characterize the chemical composition of EOs and extracts from rose petals and rose geranium, and test their efficacy against E. coli, S. aureus, S. typhimurium, A. niger, and Adenovirus 35 in different concentrations. Their toxicity levels were determined, to be used as food preservatives without causing toxicity problems in the human body. Finally, their life span was determined to establish implementation of $\mathrm{EO}$ and extract constituents as natural food preservatives.

\section{Materials and Methods}

\subsection{Essential Oils and Extracts}

Petals of Rosa $x$ damascena Herrm. (rose petals) originated from Agios Georgios Sikousis, Chios island (Northern Aegean, Greece) and were collected in May-June 2019, while Pelargonium graveolens (Thunb.) L'Hèr (rose geranium) was collected from a cultivation from the area of Patras (Achaia, Peloponnese, Greece) in May-June 2019.

The EOs were isolated with water steam distillation in an experimental $10 \mathrm{~L}$ distillery. Specifically, $0.6 \mathrm{~kg}$ of geranium leaves and $0.56 \mathrm{~kg}$ of rose petals were distilled in a final volume of $8 \mathrm{~L}$ for $3-4 \mathrm{~h}$.

The herbal extracts were produced by maceration. The extraction took place in glass and stainless-steel containers. Fresh rose petals were dried for 1 day and $2 \mathrm{~kg}$ of plant material were extracted for 35 days, at a temperature of $22-27^{\circ} \mathrm{C}$, in $40 \mathrm{~L}$ of water containing $15 \% \mathrm{v} / \mathrm{v}$ ethanol. Fresh geranium leaves were dried for $8 \mathrm{~h}$ and then $0.2 \mathrm{~kg}$ of plant material were extracted for 30 days, at a temperature of $22-27^{\circ} \mathrm{C}$, in $40 \mathrm{~L}$ of $38 \% v / v$ aqueous ethanol.

\subsection{Gas Chromatography-Mass Spectrometry}

All samples were analyzed using an Agilent Technologies 6890N equipped with a 5975B mass selective detector (MSD) in the electron impact (EI) mode of $70 \mathrm{eV}$. The capillary column was HP-5MS $(30 \mathrm{~m} \times 0.25 \mathrm{~mm}, 0.25 \mu \mathrm{m})$ with helium as a carrier gas. Data analysis was performed with GC/MSD Chemstation (Agilent Technologies Inc., Santa Clara, CA, USA) and Mestre Nova v.6.0.2-5475 (Mestrelab Research S.L., Santiago de Compostela, Spain).

The analysis of $P$. graveolens leaf EO was performed according to Sharopov et al. [19], with small modifications. In brief, the initial GC oven temperature was $56{ }^{\circ} \mathrm{C}$ for 2 min and then ramped at $3{ }^{\circ} \mathrm{C} / \mathrm{min}$ to $200^{\circ} \mathrm{C}$, and finally at $2{ }^{\circ} \mathrm{C} / \mathrm{min}$ to $220^{\circ} \mathrm{C}$ for $3 \mathrm{~min}$. Carrier gas was at a rate of $1.0 \mathrm{~mL} / \mathrm{min}$ in a splitless mode and the $m / z$ range was $35-400$. 
For the $R$. damascena petals' EO analysis, the initial GC oven temperature was $50{ }^{\circ} \mathrm{C}$ for $3 \mathrm{~min}$, which was then ramped at $5^{\circ} \mathrm{C} / \mathrm{min}$ to $100{ }^{\circ} \mathrm{C}$ for $3 \mathrm{~min}$, increased to $150{ }^{\circ} \mathrm{C}$ at a rate of $3{ }^{\circ} \mathrm{C} / \mathrm{min}$ and was held constant for $1 \mathrm{~min}$. Finally, the oven reached the temperature of $280{ }^{\circ} \mathrm{C}$ at a rate of $12^{\circ} \mathrm{C} / \mathrm{min}$ and then was kept at $280{ }^{\circ} \mathrm{C}$ for $3 \mathrm{~min}$. Carrier gas was at a rate of $1.0 \mathrm{~mL} / \mathrm{min}$, in a splitless mode and the $\mathrm{m} / \mathrm{z}$ range was $40-1000$.

All samples were diluted (1:40 for rose petals EO and 1:30 for geranium leaf EO) in ethyl acetate and the injection volume was $1 \mu \mathrm{L}$. n-Octane (98\% purity) was used as an internal standard (final concentration $0.3 \mathrm{mg} / \mathrm{mL}$ ). Alkanes (C8-C24) were analyzed under the same conditions and were used as reference points for the calculation of retention indices with the Van den Dool and Kratz equation [20]. Identification of the chemical components was based on comparison of the experimental retention indices (AIexp) and the obtained MS spectra to commercial databases [21,22] and the literature. Results were expressed as the percentage of the ratio of each compound peak area to that of the internal standard, using the program WSEARCH32 (Ver. 16/2005). Only compounds with peak area higher than $0.1 \%$ are presented.

\subsection{Liquid Chromatography-Mass Spectrometry}

The samples for UPLC-ESI-MS were prepared by dilution from the original concentrations of the extracts to a final volume of $500 \mu \mathrm{L}$. In detail, the initial geranium leaves' extract, $5.00 \mathrm{mg} / \mathrm{mL}$, was diluted to a final concentration of $4.75 \mathrm{mg} / \mathrm{mL}$, and the rose petals' extract was diluted to a final concentration of $25 \mathrm{mg} / \mathrm{mL}$ from the original $50 \mathrm{mg} / \mathrm{mL}$. Both samples included $50 \mu \mathrm{L} \mathrm{1 \%}$ formic acid in a final volume of $500 \mu \mathrm{L}$.

The single quadrupole LC/MS system of LC/MSD1260 Infinity II (Agilent Technologies Inc., Santa Clara, CA, USA) was used in this study. This system was equipped with an ESI ion source and the mass range was $m / z 100-1000$. Nitrogen was applied as gas for ionization. Separation was performed on a Poroshell 120 EC 18 column $(4.6 \times 100 \mathrm{~mm}$, $2.7 \mu \mathrm{m})$ (Agilent Technologies Inc.). LC conditions were as follows: solvent A ( $0.1 \%$ formic acid) and solvent $\mathrm{B}$ (acetonitrile with $0.1 \%$ formic acid). A gradient elution was used as follows: $0-5 \min 4 \% \mathrm{~B} ; 5-15 \min 4-15 \% \mathrm{~B} ; 15-18 \mathrm{~min} 15 \% \mathrm{~B} ; 18-23 \mathrm{~min} 15-20 \%$ B; $23-33 \min 20 \% \mathrm{~B}$; $33-48 \mathrm{~min} 20-58 \% \mathrm{~B}$; $48-63 \mathrm{~min} 58 \% \mathrm{~B}$; $63-75 \mathrm{~min} 58-95 \% \mathrm{~B}$; $75-80 \mathrm{~min} 95 \% \mathrm{~B} ; 80-84 \mathrm{~min} 95-4 \% \mathrm{~B} ; 84-88 \mathrm{~min} 4 \% \mathrm{~B}$. Flow rate was $0.5 \mathrm{~mL} / \mathrm{min}$ and the injection volume was $20 \mu \mathrm{L}$.

The standards that were used for identification were rutin (HPLC > 99\%) from Extrasynthese (Genay, France), quercetin 3-O-glucoside (HPLC > 98\%) from Extrasynthese (Genay, Gedex), and kaempferol (HPLC > 96\%) from Sigma Aldrich (Steinheim, Germany). The identification of the other compounds was based on comparison of their retention time and their mass spectra to the literature. The quantification analysis of the compounds was based on the rutin standard curve $\left(3.125-100.000 \mu \mathrm{g} / \mathrm{mL}, \mathrm{y}=29361 \mathrm{x}+425743, \mathrm{R}^{2}=0.9632\right)$. The Lower Limit of Quantitation (LLOQ) was $3.125 \mu \mathrm{g} / \mathrm{mL}$ since it is the lowest concentration of rutin giving an acceptable accuracy (relative error $<20 \%$ ) and the Lower Limit of Detection (LLOD) was $0.875 \mu \mathrm{g} / \mathrm{mL}$ calculated as a signal to noise ratio of 3 .

\subsection{Mutagenicity Assay}

The toxicity of the samples was determined by Ames Salmonella/mutagenicity assay (Salmonella test, Ames test), (EBPI, AirMetal, Los Angeles, CA, USA). The Ames test is a short-term bacterial reverse mutation trial especially designed to track a variance of chemicals that can cause genetic damage that conducts to gene mutations $[23,24]$.

The Salmonella tester strains were TA98 and TA100. TA98 causes frameshifts [25], while TA100 provokes base-pair substitution [26]. The lyophilized strains $(2.5 \mu \mathrm{L})$ were transferred to Growth Media containing $5 \mathrm{~mL}, 0.01 \mathrm{~mL}$ of Express Reagent ' $\mathrm{V}^{\prime}$, (different vial for each strain). Then, they were incubated at $37^{\circ} \mathrm{C}$ for $18 \mathrm{~h}$. The next day, the bacterial growth was checked for turbidity. The experimental procedure was continued only if there was turbidity [27]. 
The test was performed by the pre-incubation method [23]. From each colony cultivated overnight, $0.1 \mathrm{~mL}$ is taken and mixed with $0.1 \mathrm{~mL}$ of the potential mutant at various concentrations. For the EOs, the concentrations were up to $5 \%$, while for the extracts up to $90 \%$, since these are the usual upper limits of their presence in foods and drinks. Then, $2 \mathrm{~mL}$ Top Agar with 10\% sterile $0.5 \mathrm{mM}$ L-histidine $\mathrm{HCl}, 0.5 \mathrm{mM}$ biotin solution were added. The contents of each tube were poured into a sterile multi-channel reagent container and $200 \mu \mathrm{L}$ of dilutions were distributed to each of the 24-well plates, using a multi-channel pipette. After incubation at $37^{\circ} \mathrm{C}$ for $48 \mathrm{~h}$, toxicity was determined. The samples were placed in closed bags, as it is proposed for testing highly volatile chemicals and gases [25,28-30]. $S$. typhimurium cannot grow in the absence of histidine [31,32]. If developed, it means that the substance is mutagenic. The EOs are dissolved in DMSO [33,34], while the extract samples are dissolved in distilled water. The determination of toxicity was colorimetric at $600 \mathrm{~nm}$. A color change from purple to yellow signifies mutagenicity. All experiments were performed in triplicate at each concentration.

\subsection{Antibacterial Assay}

For the antibacterial activity, E. coli NCTC 9001, S. Typhimurium NCTC 12023, and S. aureus NCTC 6571 (SIGMA-ALDRICH) were grown on Brain Heart Infusion Broth (BHI Broth), (OXOID), at $37^{\circ} \mathrm{C}$ for $24 \mathrm{~h}$. As for the antifungal activity, A. niger (SIGMAALDRICH) was grown on Potato Dextrose Agar (PDA), (BIOlab), at $22{ }^{\circ} \mathrm{C}$ for 5 days [35].

The assay was performed by the agar dilution technique against a group of bacterial strains, as recommended by the National Committee for Clinical Laboratory Standards [36].

After placing $5 \mu \mathrm{L}$ of different dilutions of the EOs $\left(1, \frac{1}{2}, \frac{1}{4}\right)$ and the extracts $(90 \%, 50 \%$, 25\%) on Brain Heart Infusion Agar (BHI) plates, plates were dried at room temperature. Then, $1 \mu \mathrm{L}$ of the bacterial strain $\left(10^{8} \mathrm{CFU} / \mathrm{mL}\right)$, in serial dilutions, was spread to the plates. Plates were left to dry at room temperature for $30 \mathrm{~min}$, before the incubation. The incubation was implemented at $37^{\circ} \mathrm{C}$ for $24 \mathrm{~h}$. After incubation, there was a record of the presence or absence of microorganisms and a comparison with Control. All experiments were performed in triplicate at each concentration.

\subsection{Antifungal Assay}

After A. niger was developed on Potato Dextrose Agar (PDA), the fungal cultures were replaced on PDA that contained $5 \mu \mathrm{L}$ of different dilutions of the EOs $\left(1, \frac{1}{2}, \frac{1}{4}\right.$ dilutions) and the extracts $\left(1 / 1.1, \frac{1}{2}, \frac{1}{4}\right.$ dilutions). The plates were incubated at $22{ }^{\circ} \mathrm{C}$ for 7 days. Then, a daily monitoring of the diameter of each colony occurred and the results were recorded and compared with the Control. All experiments were performed in triplicate at each concentration. In the evaluation of the antifungal activity, the percentage inhibition was calculated.

\subsection{Antiviral Assay}

A549 cell culture (Life Science Chemilab, Athens, Greece), P +92 generation, were grown in $25 \mathrm{~cm}^{2}$ flask containing 40-50 mL Dulbecco's Minimum Essential Medium (DMEM) containing 10\% Fetal bovine serum (FBS) and 1\% antibiotic antimycotic solution. Then, cells were transferred in 24-well cell culture microplates. Fetal bovine serum was used for the growth of cells [37]. The antibiotic was added in order to avoid contamination. The cell cultures were maintained in a humidified atmosphere, $5 \% \mathrm{CO}_{2}$ at $37^{\circ} \mathrm{C}$.

Stock human Adenovirus serotype 35 was propagated in A549 cells. For titration of viruses, A549 cells were seeded in 24-well culture microplates and then incubated. Serial dilutions of virus were prepared in culture mediums. Each dilution was added into four of the wells. After incubation the cytopathic effect in each well was recorded.

First of all, the cytopathic effect of the samples was performed with the same process as the cytopathic effect of Adenovirus. Then, the analysis of the antiviral activity of EOs and extracts took place. Cell line A549 was incubated overnight in 12-well plates at $37^{\circ} \mathrm{C}$ with $5 \% \mathrm{CO}_{2}$, until cells got "confluent $90-100 \%$ " overlap. The volume of each cell line 
on each well plate was calculated by counting the number of cells at the four corners of the Neubauer.

Then solutions containing serial concentrations of $\mathrm{AdV}(30 \mu \mathrm{L})$ and the sample $(30 \mu \mathrm{L})$ were added and incubated with stirring $(150 \mathrm{rpm})$ for $90 \mathrm{~min}$ at $37^{\circ} \mathrm{C}$. The medium was discarded and DMEM was re-added with $1 \%$ FBS. The 12 -well plates were incubated for $3-4$ days at $37^{\circ} \mathrm{C}$ with $5 \% \mathrm{CO}_{2}$. Subsequently, the cytotoxicity was observed by electron microscope. Tests were performed according to Saderi et al., 2011 [28], claiming that the concentrations of the samples that entirely suspend AdV35 cytopathic effect, is recorded as efficient concentration, comparing to virus control. All experiments were performed in duplicate at each concentration.

\subsection{Life Span Assay}

To determine the life span of the samples, samples were added to the proper medium. Samples were added to the Tryptone Bile X-Glucuronide Medium (TBX), to detect $E$. coli. Also, samples were added to the Rappaport Vassiliadis broth (RVS), to detect Salmonella spp. Furthermore, samples were added to the Baird-Parker Agar (BP), (OXOID), in order to detect $S$. aureus, and finally, were added to the Potato Dextrose Agar (PDA), (BIOlab), to detect $A$. niger.

In order to determine the time when the consumption of the products is safe, a threeweek procedure was performed. The samples were kept in appropriate conditions, in the refrigerator at $10^{\circ} \mathrm{C}$ for a period of five weeks, before the experimental procedure began. The experimental procedure was carried out for a period of three weeks, when the samples were stored in a refrigerator at $10^{\circ} \mathrm{C}$. All experiments were performed in duplicate at each concentration.

\section{Results}

\subsection{Chemical Analysis}

The chemical analysis of the metabolites of EOs and extracts is presented in the Tables 1-3. The evaluation of the mass spectra of each chromatographic peak did not allude to the presence of any pesticide or other contaminants in the samples.

Table 1. Volatile metabolites in essential oils of the geranium leaves and rose petals.

\begin{tabular}{|c|c|c|c|c|}
\hline \multirow[t]{2}{*}{ Components } & \multirow[t]{2}{*}{$\operatorname{AIexp}^{a}$} & \multirow[t]{2}{*}{ AItheor $^{b}$} & \multicolumn{2}{|c|}{$\%$ Peak Area/IS Area } \\
\hline & & & Pelargonium graveolens & Rosa damascena \\
\hline$\alpha$-pinene & 929 & 932 & $0.19 \pm 0.02$ & nd \\
\hline cis-linalool oxide (fr) & 1069 & 1067 & $0.21 \pm 0.08$ & nd \\
\hline trans-linalool oxide (fr) & 1085 & 1084 & $0.17 \pm 0.03$ & nd \\
\hline linalool & 1092 & 1095 & $2.93 \pm 0.17$ & $3.32 \pm 0.17$ \\
\hline cis-rose oxide & 1108 & 1106 & $0.84 \pm 0.06$ & nd \\
\hline phenyl ethyl alcohol & 1117 & 1107 & nd & $40.03 \pm 0.13$ \\
\hline trans-rose oxide & 1125 & 1122 & $0.29 \pm 0.01$ & nd \\
\hline menthone & 1150 & 1148 & $0.41 \pm 0.06$ & nd \\
\hline isomenthone & 1163 & 1158 & $3.02 \pm 0.09$ & $0.41 \pm 0.06$ \\
\hline$\alpha$-terpineol & 1195 & 1186 & $0.16 \pm 0.00$ & $0.74 \pm 0.01$ \\
\hline methyl chavicol (estragole) & 1205 & 1195 & nd & $0.59 \pm 0.00$ \\
\hline citronellol & 1228 & 1223 & $26.73 \pm 1.65$ & $19.89 \pm 0.34$ \\
\hline neral & 1239 & 1235 & $0.17 \pm 0.00$ & nd \\
\hline geraniol & 1254 & 1249 & $10.1 \pm 0.17$ & $27.8 \pm 0.90$ \\
\hline citronellyl formate & 1274 & 1271 & $3.96 \pm 0.01$ & $0.76 \pm 0.27$ \\
\hline geranyl formate & 1300 & 1298 & $1.05 \pm 0.06$ & nd \\
\hline citronellyl acetate & 1352 & 1350 & $0.14 \pm 0.00$ & nd \\
\hline$\alpha$-copaene & 1371 & 1374 & $0.33 \pm 0.07$ & nd \\
\hline$\beta$-bourbonene & 1380 & 1387 & $0.81 \pm 0.03$ & nd \\
\hline geranyl acetate & 1383 & 1379 & $0.16 \pm 0.02$ & nd \\
\hline$\beta$-elemene & 1393 & 1389 & nd & $1.10 \pm 0.00$ \\
\hline vanillin & 1399 & 1393 & $7.06 \pm 1.79$ & nd \\
\hline
\end{tabular}


Table 1. Cont.

\begin{tabular}{|c|c|c|c|c|}
\hline \multirow[t]{2}{*}{ Components } & \multirow[t]{2}{*}{$\operatorname{AIexp}^{a}$} & \multirow[t]{2}{*}{ AItheor ${ }^{b}$} & \multicolumn{2}{|c|}{$\%$ Peak Area/IS Area } \\
\hline & & & Pelargonium graveolens & Rosa damascena \\
\hline E-caryophyllene & 1414 & 1417 & $1.12 \pm 1.25$ & $0.82 \pm 0.00$ \\
\hline aromadendrene & 1439 & 1439 & $0.31 \pm 0.07$ & nd \\
\hline citronellyl propanoate & 1442 & 1444 & $0.22 \pm 0.01$ & nd \\
\hline allo-aromadendrene & 1456 & 1458 & $\mathrm{nq}$ & nd \\
\hline geranyl propanoate & 1473 & 1476 & $0.46 \pm 0.05$ & nd \\
\hline$\gamma$-muurolene & 1477 & 1480 & nd & $0.28 \pm 0.05$ \\
\hline E- $\beta$-ionone & 1489 & 1487 & nd & $0.50 \pm 0.26$ \\
\hline$\alpha$-muurolene & 1501 & 1500 & $0.14 \pm 0.00$ & $0.28 \pm 0.04$ \\
\hline$\gamma$-cadinene & 1509 & 1513 & $0.16 \pm 0.00$ & nd \\
\hline$\delta$-cadinene & 1519 & 1522 & $0.74 \pm 0.48$ & nd \\
\hline citronellyl butanoate & 1526 & 1530 & $0.39 \pm 0.01$ & nd \\
\hline$\alpha$-agarofuran & 1540 & 1548 & $0.30 \pm 0.14$ & nd \\
\hline geranyl butanoate & 1559 & 1562 & $0.49 \pm 0.05$ & nd \\
\hline spathulenol & 1573 & 1577 & $\mathrm{nq}$ & nd \\
\hline caryophyllene oxide & 1578 & 1582 & $0.24 \pm 0.00$ & nd \\
\hline phenyl ethyl tiglate & 1584 & 1584 & $0.34 \pm 0.03$ & nd \\
\hline 10-epi- $\gamma$-eudesmol & 1614 & 1622 & $3.28 \pm 0.38$ & nd \\
\hline$\gamma$-eudesmol & 1632 & 1630 & $0.25 \pm 0.00$ & nd \\
\hline $\begin{array}{l}\text { 4a-hydroxy-dihydro } \\
\text { agarofuran }\end{array}$ & 1643 & 1651 & $0.28 \pm 0.00$ & nd \\
\hline geranyl tiglate & 1700 & 1696 & $0.74 \pm 0.15$ & nd \\
\hline hexadecanoic acid & 1961 & 1959 & $0.65 \pm 0.00$ & nd \\
\hline $\begin{array}{l}\text { number of components } \\
\text { total identified }\end{array}$ & & & $\begin{array}{c}39 \\
67.98 \pm 5.15\end{array}$ & $\begin{array}{c}13 \\
96.47 \pm 0.43\end{array}$ \\
\hline
\end{tabular}

Notes: IS: internal standard, nd: not detected, nq: not quantified. Results are presented as mean \pm standard deviation derived from triplicate analysis. ${ }^{a}$ Retention index on an apolar HP-5MS column. ${ }^{b}$ Literature retention indices on apolar column from Adams et al., 2012 [21].

Table 2. LC/MS identification of metabolites and their concentration in the extract of rose petals.

\begin{tabular}{|c|c|c|c|c|c|c|c|}
\hline Peak & Rt (min) & Negative Ionization $(\mathrm{m} / \mathrm{z})$ & Positive Ionization $(\mathrm{m} / \mathrm{z})$ & M.W. & $\begin{array}{l}\text { Molecular } \\
\text { Formula }\end{array}$ & Tentative Identification & $\mathrm{C}(\mu \mathrm{g} / \mathrm{mL})$ \\
\hline 1 & 1.8 & $\begin{array}{c}179[\mathrm{M}-\mathrm{H}]^{-} \\
215[\mathrm{M}+\mathrm{Cl}]^{-} \\
217[\mathrm{M}+\mathrm{K}-2 \mathrm{H}]^{-}\end{array}$ & $\begin{array}{c}203[\mathrm{M}+\mathrm{Na}]^{+} \\
383[2 \mathrm{M}+\mathrm{Na}]^{+}\end{array}$ & 180 & $\mathrm{C}_{6} \mathrm{H}_{12} \mathrm{O}_{6}$ & Hexose $^{52}$ & $5.3 \pm 0.4$ \\
\hline 2 & 1.9 & $\begin{array}{c}341[\mathrm{M}-\mathrm{H}]^{-} \\
683[2 \mathrm{M}-\mathrm{H}]^{-} \\
161[\mathrm{M}-\mathrm{H}-180 \text { (hexose) }]^{-}\end{array}$ & $365[\mathrm{M}+\mathrm{Na}]^{+}$ & 342 & $\mathrm{C}_{15} \mathrm{H}_{18} \mathrm{O}_{9}$ & Caffeoyl hexoside ${ }^{49}$ & nq \\
\hline 3 & 25.4 & $\begin{array}{c}609[\mathrm{M} \mathrm{-} \mathrm{H}]^{-} \\
301[\text { Quercetin - H] }]^{-}\end{array}$ & $\begin{array}{c}611[\mathrm{M}+\mathrm{H}]^{+} \\
325[\mathrm{M}+\mathrm{H}+\mathrm{K}]^{2+} \\
633[\mathrm{M}+\mathrm{Na}]^{+}\end{array}$ & 610 & $\mathrm{C}_{27} \mathrm{H}_{30} \mathrm{O}_{16}$ & $\begin{array}{l}\text { Rutin (Quercetin } \\
\text { 3-O-rutinoside) st }\end{array}$ & $\mathrm{nq}$ \\
\hline 4 & 25.9 & $\begin{array}{c}463[\mathrm{M} \mathrm{-} \mathrm{H}]^{-} \\
928[2 \mathrm{M}-\mathrm{H}]^{-} \\
\text {300[Quercetin - 2H] } \\
\text { 463[M - H] }]^{-}\end{array}$ & $\begin{array}{c}465[\mathrm{M}+\mathrm{H}]^{+} \\
487[\mathrm{M}+\mathrm{Na}]^{+} \\
952[2 \mathrm{M}+\mathrm{Na}]^{+} \\
465[\mathrm{M}+\mathrm{H}]^{+}\end{array}$ & 464 & $\mathrm{C}_{21} \mathrm{H}_{20} \mathrm{O}_{12}$ & Quercetin-3-O-hexoside ${ }^{48,49,50}$ & $7.0 \pm 0.3$ \\
\hline 5 & 26.4 & $\begin{array}{c}928[2 \mathrm{M}-\mathrm{H}]^{-} \\
301[\text { Quercetin - H] }]^{-}\end{array}$ & $\begin{array}{c}487[\mathrm{M}+\mathrm{Na}]^{+} \\
952[2 \mathrm{M}+\mathrm{Na}]^{+}\end{array}$ & 464 & $\mathrm{C}_{21} \mathrm{H}_{20} \mathrm{O}_{12}$ & Quercetin-3-O-glucoside ${ }^{\text {st }}$ & $7.4 \pm 0.2$ \\
\hline 6 & 27.9 & $\begin{array}{c}\text { 593[M - H] }]^{-} \\
\text {285[Kaempferol - H] }]^{-}\end{array}$ & $\begin{array}{c}595[\mathrm{M}+\mathrm{H}]^{+} \\
317[\mathrm{M}+\mathrm{H}+\mathrm{K}]^{2+} \\
617[\mathrm{M}+\mathrm{Na}]^{+}\end{array}$ & 594 & $\mathrm{C}_{27} \mathrm{H}_{30} \mathrm{O}_{15}$ & Kaempferol disaccharide ${ }^{48,50}$ & $\mathrm{nq}$ \\
\hline 7 & 28.1 & $\begin{array}{c}447[\mathrm{M} \mathrm{-} \mathrm{H}]^{-} \\
896[2 \mathrm{M}-\mathrm{H}]^{-} \\
285[\text { Kaempferol - H] }]^{-}\end{array}$ & $\begin{array}{c}449[\mathrm{M}+\mathrm{H}]^{+} \\
471[\mathrm{M}+\mathrm{Na}]^{+} \\
920[2 \mathrm{M}+\mathrm{Na}]^{+} \\
611[\mathrm{M}+\mathrm{H}]^{+}\end{array}$ & 448 & $\mathrm{C}_{21} \mathrm{H}_{20} \mathrm{O}_{11}$ & Kaempferol hexoside $48,49,50$ & $14.7 \pm 1.5$ \\
\hline 8 & 28.5 & $609[\mathrm{M}-\mathrm{H}]^{-}$ & $\begin{array}{c}325[\mathrm{M}+\mathrm{H}+\mathrm{K}]^{2+} \\
633[\mathrm{M}+\mathrm{Na}]^{+}\end{array}$ & 610 & $\mathrm{C}_{27} \mathrm{H}_{30} \mathrm{O}_{16}$ & Quercetin disaccharide ${ }^{50}$ & $\mathrm{nq}$ \\
\hline 9 & 28.9 & $433[\mathrm{M}-\mathrm{H}]^{-}$ & $\begin{array}{c}435[\mathrm{M}+\mathrm{H}]^{+} \\
457[\mathrm{M}+\mathrm{Na}]^{+}\end{array}$ & 434 & $\mathrm{C}_{20} \mathrm{H}_{18} \mathrm{O}_{11}$ & Quercetin-3-O-arabinoside ${ }^{51}$ & $\mathrm{nq}$ \\
\hline 10 & 29.3 & $\begin{array}{c}447[\mathrm{M}-\mathrm{H}]^{-} \\
896[2 \mathrm{M}-\mathrm{H}]^{-} \\
285[\mathrm{M}-\mathrm{H}-163(\text { hexose })]^{-}\end{array}$ & $\begin{array}{c}449[\mathrm{M}+\mathrm{H}]^{+} \\
471[\mathrm{M}+\mathrm{Na}]^{+} \\
920[2 \mathrm{M}+\mathrm{Na}]^{+}\end{array}$ & 448 & $\mathrm{C}_{21} \mathrm{H}_{20} \mathrm{O}_{11}$ & Kaempferol-3-O-glucoside ${ }^{49,51}$ & $89.0 \pm 0.5$ \\
\hline 11 & 31.5 & $435[\mathrm{M}-\mathrm{H}]^{-}$ & $\begin{array}{c}459[\mathrm{M}+\mathrm{Na}]^{+} \\
238[\mathrm{M}+\mathrm{H}+\mathrm{K}]^{2+} \\
419[\mathrm{M}+\mathrm{H}]^{+}\end{array}$ & 436 & & Unknown & $\mathrm{nq}$ \\
\hline 12 & 31.7 & $417[\mathrm{M}-\mathrm{H}]^{-}$ & $\begin{array}{c}441[\mathrm{M}+\mathrm{Na}]^{+} \\
895[2 \mathrm{M}+\mathrm{Na}]^{+}\end{array}$ & 418 & $\mathrm{C}_{20} \mathrm{H}_{18} \mathrm{O}_{10}$ & Kaempferol pentoside $49,50,51$ & $\mathrm{nq}$ \\
\hline
\end{tabular}


Table 2. Cont.

\begin{tabular}{|c|c|c|c|c|c|c|c|}
\hline Peak & Rt (min) & Negative Ionization $(\mathrm{m} / \mathrm{z})$ & Positive Ionization $(\mathrm{m} / \mathrm{z})$ & M.W. & $\begin{array}{l}\text { Molecular } \\
\text { Formula }\end{array}$ & Tentative Identification & $C(\mu \mathrm{g} / \mathrm{mL})$ \\
\hline 13 & 33.1 & $\begin{array}{c}593[\mathrm{M} \mathrm{-} \mathrm{H}]^{-} \\
285\left[^{-} \text {Kaempferol - H }\right]^{-}\end{array}$ & $\begin{array}{c}595[\mathrm{M}+\mathrm{H}]^{+} \\
317[\mathrm{M}+\mathrm{H}+\mathrm{K}]^{2+} \\
617[\mathrm{M}+\mathrm{Na}]^{+}\end{array}$ & 594 & $\mathrm{C}_{27} \mathrm{H}_{30} \mathrm{O}_{15}$ & $\begin{array}{l}\text { Kaempferol disaccharide } \\
\text { (Kaempferol -O-pentose } \\
\text {-O-glucuronic acid) } 48,49,50,51\end{array}$ & $19.7 \pm 1.5$ \\
\hline 14 & 33.9 & $\begin{array}{c}417[\mathrm{M}-\mathrm{H}]^{-} \\
836[2 \mathrm{M}-\mathrm{H}]^{-} \\
285[\mathrm{M}-\mathrm{H}-133 \\
(\text { pentose })]^{-}\end{array}$ & $\begin{array}{c}419[\mathrm{M}+\mathrm{H}]^{+} \\
441[\mathrm{M}+\mathrm{Na}]^{+}\end{array}$ & 418 & $\mathrm{C}_{20} \mathrm{H}_{18} \mathrm{O}_{10}$ & Kaempferol pentoside ${ }^{49,51}$ & $\mathrm{nq}$ \\
\hline 15 & 35.9 & $\begin{array}{c}431[\mathrm{M}-\mathrm{H}]^{-} \\
863[2 \mathrm{M}-\mathrm{H}]^{-}\end{array}$ & $\begin{array}{c}433[\mathrm{M}+\mathrm{H}]^{+} \\
455[\mathrm{M}+\mathrm{Na}]^{+} \\
888[2 \mathrm{M}+\mathrm{Na}]^{+}\end{array}$ & 432 & $\mathrm{C}_{21} \mathrm{H}_{20} \mathrm{O}_{10}$ & Kaempferol deoxyhexoside ${ }^{50}$ & $17.5 \pm 1.5$ \\
\hline 16 & 40.8 & $\begin{array}{c}\text { 635[M - H }]^{-} \\
593[\text { Kaempferol } \\
\text { disaccharide - H }]^{-}\end{array}$ & $\begin{array}{c}637[\mathrm{M}+\mathrm{H}]^{+} \\
659[\mathrm{M}+\mathrm{Na}]^{+} \\
338[\mathrm{M}+\mathrm{H}+\mathrm{K}]^{+}\end{array}$ & 636 & $\mathrm{C}_{29} \mathrm{H}_{32} \mathrm{O}_{16}$ & Kaempferol acetyldisaccharide & $\mathrm{nq}$ \\
\hline 17 & 41.7 & $593[\mathrm{M}-\mathrm{H}]^{-}$ & $\begin{array}{c}595[\mathrm{M}+\mathrm{H}]^{+} \\
617[\mathrm{M}+\mathrm{Na}]^{+}\end{array}$ & 594 & $\mathrm{C}_{27} \mathrm{H}_{30} \mathrm{O}_{15}$ & $\begin{array}{c}\text { Kaempferol disaccharide } \\
\text { (Kaempferol-O-hexose-O- } \\
\text { deoxyhexose) } \\
50,53\end{array}$ & $\mathrm{nq}$ \\
\hline 18 & 44.7 & $285[\mathrm{M}-\mathrm{H}]^{-}$ & $287[\mathrm{M}+\mathrm{H}]^{+}$ & 286 & $\mathrm{C}_{15} \mathrm{H}_{10} \mathrm{O}_{6}$ & Kaempferol $^{\text {st }}$ & $\mathrm{nq}$ \\
\hline
\end{tabular}

Notes nq: not quantified. st: standard compound used for identification. The superscript numbers indicate the previous studies on Rosa spp. that report the same ingredient.

Table 3. LC/MS identification of metabolites and their concentration in the extract of rose geranium leaves.

\begin{tabular}{|c|c|c|c|c|c|c|c|}
\hline Peak & $\begin{array}{c}\mathrm{Rt} \\
(\mathrm{min})\end{array}$ & Negative Ionization $(\mathrm{m} / \mathrm{z})$ & Positive Ionization $(\mathrm{m} / \mathrm{z})$ & M.W. & $\begin{array}{l}\text { Molecular } \\
\text { Formula }\end{array}$ & Tentative Identification & $C(\mu \mathrm{g} / \mathrm{mL})$ \\
\hline 1 & 23.2 & $\begin{array}{c}595[\mathrm{M}-\mathrm{H}]^{-} \\
462[\mathrm{M}-\mathrm{H}-132]^{-} \\
445\left[\mathrm{M}-\mathrm{H}-132-\mathrm{H}_{2} \mathrm{O}\right]^{-} \\
300[\text { quercetin - H] }]^{-}\end{array}$ & $\begin{array}{c}597[\mathrm{M}+\mathrm{H}]^{+} \\
619[\mathrm{M}+\mathrm{Na}]^{+}\end{array}$ & 596 & $\mathrm{C}_{26} \mathrm{H}_{28} \mathrm{O}_{16}$ & Quercetin-3-O-pentosyl hexoside ${ }^{54}$ & $15.7 \pm 0.2$ \\
\hline 2 & 25 & $\begin{array}{c}609[\mathrm{M} \mathrm{-} \mathrm{H}]^{-} \\
\text {301[quercetin }]^{-} \\
\text {300[quercetin - H] }]^{-} \\
179 \\
463[\mathrm{M}-\mathrm{H}]^{-}\end{array}$ & $\begin{array}{c}611[\mathrm{M}+\mathrm{H}]^{+} \\
325[\mathrm{M}+\mathrm{H}+\mathrm{K}]^{2+} \\
633[\mathrm{M}+\mathrm{Na}]^{+}\end{array}$ & 610 & $\mathrm{C}_{27} \mathrm{H}_{30} \mathrm{O}_{16}$ & $\begin{array}{l}\text { Quercetin-3-O-rhamnoside } \\
\text { hexoside }{ }^{54,55,56,57}\end{array}$ & $\mathrm{nq}$ \\
\hline 3 & 25.5 & $\begin{array}{c}\text { 927[2M - H }]^{-} \\
\text {316[Myricetin - } 2 \mathrm{H}]^{-} \\
\text {317[Myricetin - H] }]^{-} \\
\text {287, } 179\end{array}$ & $\begin{array}{c}465[\mathrm{M}+\mathrm{H}]^{+} \\
487[\mathrm{M}+\mathrm{Na}]^{+} \\
951[2 \mathrm{M}+\mathrm{Na}]^{+}\end{array}$ & 464 & $\mathrm{C}_{21} \mathrm{H}_{20} \mathrm{O}_{12}$ & Myricetin-3-O-rhamnoside ${ }^{54}$ & $\mathrm{nq}$ \\
\hline 4 & 25.8 & $\begin{array}{c}463[\mathrm{M}-\mathrm{H}]^{-} \\
927[2 \mathrm{M}-\mathrm{H}]^{-} \\
301[\text { quercetin - H] } \\
\text { 300[quercetin - 2H] }]^{-} 179\end{array}$ & $\begin{array}{c}465[\mathrm{M}+\mathrm{H}]^{+} \\
487[\mathrm{M}+\mathrm{Na}]^{+} \\
951[2 \mathrm{M}+\mathrm{Na}]^{+}\end{array}$ & 464 & $\mathrm{C}_{21} \mathrm{H}_{20} \mathrm{O}_{12}$ & Quercetin-3-O-galactoside ${ }^{54}$ & $13.9 \pm 1.2$ \\
\hline 5 & 26.3 & $\begin{array}{c}463[\mathrm{M}-\mathrm{H}]^{-} \\
927[2 \mathrm{M}-\mathrm{H}]^{-} \\
301[\text { quercetin - H] }]^{-} \\
255,179\end{array}$ & $\begin{array}{c}465[\mathrm{M}+\mathrm{H}]^{+} \\
487[\mathrm{M}+\mathrm{Na}]^{+} \\
951[2 \mathrm{M}+\mathrm{Na}]^{+}\end{array}$ & 464 & $\mathrm{C}_{21} \mathrm{H}_{20} \mathrm{O}_{12}$ & Quercetin-3-O-glucoside $^{\text {st }}$ & $17.7 \pm 0.3$ \\
\hline 6 & 27.9 & $\begin{array}{c}433[\mathrm{M}-\mathrm{H}]^{-} \\
867[2 \mathrm{M}-\mathrm{H}]^{-} \\
\text {300[quercetin - H] }]^{-} \\
255\end{array}$ & $\begin{array}{c}435[\mathrm{M}+\mathrm{H}]^{+} \\
457[\mathrm{M}+\mathrm{Na}]^{+} \\
891[2 \mathrm{M}+\mathrm{Na}]^{+}\end{array}$ & 434 & $\mathrm{C}_{20} \mathrm{H}_{18} \mathrm{O}_{11}$ & Quercetin 3-O- pentoside ${ }^{54}$ & $3.8 \pm 0.6$ \\
\hline 7 & 28 & $\begin{array}{c}447[\mathrm{M}-\mathrm{H}]^{-} \\
895[2 \mathrm{M}-\mathrm{H}]^{-} \\
285[\mathrm{M}-\mathrm{H}-163]^{-}\end{array}$ & $\begin{array}{c}449[\mathrm{M}+\mathrm{H}]^{+} \\
471[\mathrm{M}+\mathrm{Na}]^{+} \\
919[2 \mathrm{M}+\mathrm{Na}]^{+}\end{array}$ & 448 & $\mathrm{C}_{21} \mathrm{H}_{20} \mathrm{O}_{11}$ & Kaempferol 3-O-glucoside 54,55 & $4.7 \pm 0.0$ \\
\hline 8 & 29.3 & $\begin{array}{c}447[\mathrm{M}-\mathrm{H}]^{-} \\
895[2 \mathrm{M}-\mathrm{H}]^{-}\end{array}$ & $\begin{array}{c}449[\mathrm{M}+\mathrm{H}]^{+} \\
471[\mathrm{M}+\mathrm{Na}]^{+} \\
919[2 \mathrm{M}+\mathrm{Na}]^{+}\end{array}$ & 448 & $\mathrm{C}_{21} \mathrm{H}_{20} \mathrm{O}_{11}$ & Kaempferol 3-O-galactoside ${ }^{52}$ & $\mathrm{nq}$ \\
\hline 9 & 30.6 & $417[\mathrm{M}-\mathrm{H}]^{-}$ & $\begin{array}{c}419[\mathrm{M}+\mathrm{H}]^{+} \\
441[\mathrm{M}+\mathrm{Na}]^{+}\end{array}$ & 418 & $\mathrm{C}_{20} \mathrm{H}_{18} \mathrm{O}_{10}$ & Kaempferol 3-O- pentoside ${ }^{54}$ & $\mathrm{nq}$ \\
\hline 10 & 24.9 & $\begin{array}{c}\text { 507[M + Formic Acid - } \\
\mathrm{H}]^{-}\end{array}$ & $485[\mathrm{M}+\mathrm{Na}]^{+}$ & 462 & $\mathrm{C}_{21} \mathrm{H}_{18} \mathrm{O}_{12}$ & Scutelarein-7-O- $\beta$-glucuronide ${ }^{58}$ & $\mathrm{nq}$ \\
\hline 11 & 40.1 & $313[\mathrm{M}-\mathrm{H}]^{-}$ & $\begin{array}{c}315[\mathrm{M}+\mathrm{H}]^{+} \\
651[2 \mathrm{M}+\mathrm{Na}]^{+}\end{array}$ & 314 & $\mathrm{C}_{17} \mathrm{H}_{14} \mathrm{O} 6$ & Cirsimaritin $^{58}$ & $\mathrm{nq}$ \\
\hline
\end{tabular}

Notes nq: not quantified, st: standard compound used for identification. The superscript numbers indicate the previous studies on leaves of Pelargonium spp. that mention the same ingredient.

\subsection{Antimutagenesis}

Antimutagenic effect regularly depends on the dose of the sample [38]. In this study, the doses of 5\% for EOs and $90 \%$ for extracts were not toxic for the strains (Table 4). All EOs were able to inhibit mutations induced by TA98 and TA100 Salmonella strains, as well as all extracts at concentrations up to $90 \%$. 
Table 4. Results from essential oil and plant extracts toxicity experiments.

\begin{tabular}{|c|c|c|c|c|}
\hline EOs & $\begin{array}{c}\text { NEG } \\
\text { CONTROL }\end{array}$ & $\begin{array}{c}\text { Pelargonium } \\
\text { graveolens (5\%) }\end{array}$ & $\begin{array}{c}\text { Rosa damascena } \\
(5 \%)\end{array}$ & $\begin{array}{c}\text { POS } \\
\text { CONTROL }\end{array}$ \\
\hline TA98 & Nontoxic & Nontoxic & Nontoxic & Toxic \\
\hline TA100 & Nontoxic & Nontoxic & Nontoxic & Toxic \\
\hline Extract & $\begin{array}{c}\text { NEG } \\
\text { CONTROL }\end{array}$ & $\begin{array}{c}\text { Pelargonium } \\
\text { graveolens }(90 \%)\end{array}$ & $\begin{array}{c}\text { Rosa damascena } \\
(90 \%)\end{array}$ & $\begin{array}{c}\text { POS } \\
\text { CONTROL }\end{array}$ \\
\hline TA98 & Nontoxic & Nontoxic & Nontoxic & Toxic \\
\hline TA100 & Nontoxic & Nontoxic & Nontoxic & Toxic \\
\hline
\end{tabular}

\subsection{Antibacterial Activity}

The antibacterial activities of the different kinds of EOs and extracts were assessed by the agar dilution method. It can be seen from the data shown in Table 5, that all essential oils and extracts had some antibacterial activity on the tested strains, nevertheless the antibacterial properties varied significantly.

Table 5. Percentage of essential oils and extracts inhibitory activity against bacteria at different values of final content in growth medium (from 5 to $100 \%$ for essential oils and 25 to $90 \%$ for extracts).

\begin{tabular}{cccc}
\hline Essential Oils & E. coli & S. aureus & Salmonella spp. \\
\hline Pelargonium graveolens (100\%) & $85 \%$ & $55 \%$ & $51 \%$ \\
Pelargonium graveolens (50\%) & $29 \%$ & $34 \%$ & $31 \%$ \\
Pelargonium graveolens (5\%) & - & $6 \%$ & $13 \%$ \\
Rosa damascena $(100 \%)$ & $65 \%$ & $42 \%$ & $86 \%$ \\
Rosa damascena (50\%) & $36 \%$ & $40 \%$ & $73 \%$ \\
Rosa damascena (5\%) & $2 \%$ & $28 \%$ & $22 \%$ \\
\hline Extracts & & & \\
\hline Pelargonium graveolens (90\%) & $68 \%$ & $40 \%$ & $42 \%$ \\
Pelargonium graveolens (50\%) & $59 \%$ & $28 \%$ & $33 \%$ \\
Pelargonium graveolens (25\%) & $46 \%$ & $14 \%$ & $86 \%$ \\
Rosa damascena (90\%) & $74 \%$ & $68 \%$ & $65 \%$ \\
Rosa damascena (50\%) & $57 \%$ & $43 \%$ & $45 \%$ \\
Rosa damascena (25\%) & $35 \%$ & $37 \%$ & \\
\hline
\end{tabular}

Note: "-" indicates that the essential oil had no inhibitory activity on the tested strain at this concentration. 0-25\%, no or little inhibition; $26-50 \%$, average inhibition; $51-75 \%$, strong inhibition. According to the CLSI breaking points for a given inhibitor concentration: $\%$ inhibition $=100 *[1-(x-\min ) /(\max -\min )]$, Humphries et al., 2019 [39].

P. graveolens' essential oil showed a significant antibacterial activity (85\%) against E. coli in 100\% concentration. In lower concentrations, it did not affect E. coli growth. On the other hand, the extract showed good antibacterial activity (68\%) against E. coli in $90 \%$ concentration and average efficacy in 50\% and $25 \%$ concentrations (59\% and $46 \%$ respectively). As for S. aureus, the essential oil had good antibacterial activity (55\%) in $100 \%$ concentration. In lower concentrations, it was not effective. The extract was not effective against this bacterium - only in $90 \%$ concentration it appeared to have a minor inhibition (40\%). Finally, the essential oil had a small activity against Salmonella spp. (51\%), in 100\% concentration. In lower concentrations, its activity was negligible. The extract was almost efficient in $90 \%, 42 \%$, while in lower concentrations, it had no effect.

$R$. damascena's essential oil showed a noteworthy antibacterial activity against $E$. coli $(65 \%$, in $100 \%)$. The extract revealed a remarkable antibacterial activity $(74 \%$, in $90 \%$ and $57 \%$ in 50\%). Furthermore, the antibacterial activity against S. aureus, was only noticeable (68\%) in the extract in concentration 90\%. Last of all, the antimicrobial potency against Salmonella spp., showed drastic essential oil action ( $86 \%$ in $100 \%$ concentration and $73 \%$ in $50 \%$ ), but also capable extract activity ( $86 \%$ in $90 \%$ concentration, $65 \%$ in $50 \%$ and $45 \%$ in $25 \%$ ). 


\subsection{Antifungal Activity}

Antifungal activity is presented in Figure $1 \mathrm{a}-\mathrm{c}$ for $\mathrm{EO}$ and Figure $2 \mathrm{a}-\mathrm{c}$ for extracts. The Figures below Figure 1a-c show the increase (in $\mathrm{cm}$ ) in fungal diameter over the course of one week (7 days) in the presence of the essential oils at different percentages.
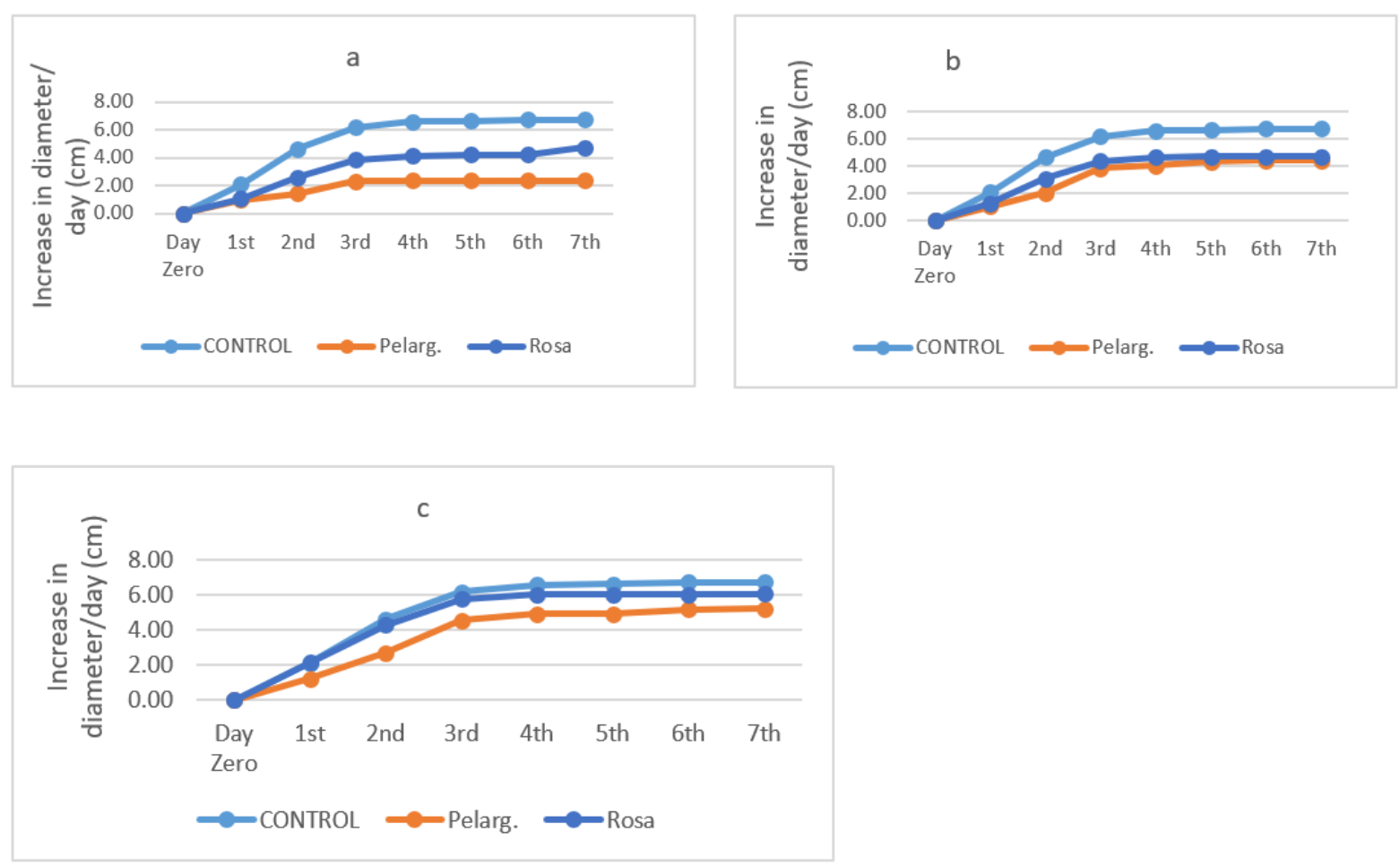

Figure 1. Antifungal activity of essential oils 100\% (a), 50\% (b), 5\% (c) through time.
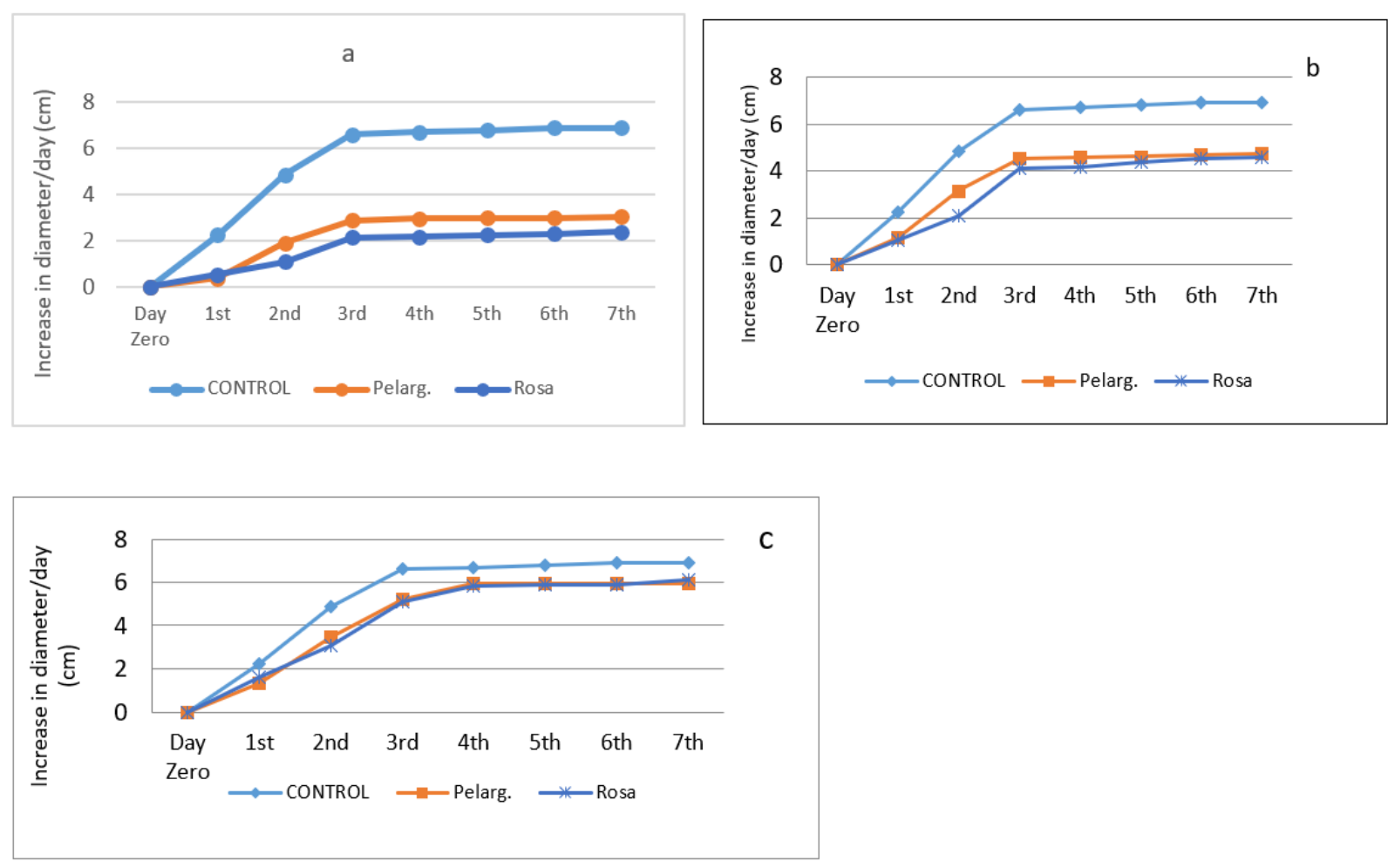

Figure 2. Antifungal activity through time of extracts at concentrations of $90 \%$ (a), $50 \%$ (b), and $25 \%$ (c). 
Experimental data show that all EOs in the concentrations of $100 \%$ and $50 \%$ were particularly effective against the fungus. At the end of the third day, the fungus stabilizes almost in diameter in all the oils and stops growing, while in the control it grows until it occupies the whole petri dish, which is why in the last days of the experiment its growth rate is low. As the days go by, the growth rate reaches saturation. The reason this happens is because natural preservatives contain nutrients that initially favor the growth of the fungus. Nevertheless, this particular resistance can disappear [40]. The EO of rose geranium has the greatest effect on the fungus. At lower concentrations rose geranium EO, even at a concentration of $5 \%$, maintained activity. Figure 2 presents the increase (in $\mathrm{cm}$ ) in fungal diameter over the course of one week (7 days) for P. graveolens' and $R$. damascena' extracts.

From the above experimental data, it appears that the $90 \%$ extracts were efficient. In this case also, the fungi grow until the third day and then their growth stops, while in the control sample the growth is limited by the petri dish and stops. Potato Dextrose Agar (PDA) provides a nutrient base for luxuriant growth of most fungi [41]. In 50\% plant extracts, they have a moderate effect whereas in the $25 \%$, plant extracts have similar effect.

\subsection{Antiviral Activity}

The results of cytotoxicity assay for tested samples are shown in Table 6 . The cytopathic effects for EOs were observed in concentrations up to $5 \%$, while the extracts did not show cytopathic effect even at $100 \%$ concentration.

Table 6. Effects of cytotoxicity of essential oils.

\begin{tabular}{cccc}
\hline Essential Oil & Effect on Cell Line A549 & Extract & Effect on Cell Line A549 \\
\hline Pelargonium graveolens $100 \%$ & Cytotoxic & Pelargonium graveolens $100 \%$ & Non-cytotoxic \\
\hline Pelargonium graveolens $5 \%$ & Noncytotoxic & Pelargonium graveolens $90 \%$ & Non-cytotoxic \\
\hline Rosa damascena $100 \%$ & Cytotoxic & Rosa damascena $100 \%$ & Non-cytotoxic \\
\hline Rosa damascena $5 \%$ & Noncytotoxic & Rosa damascena $90 \%$ & Non-cytotoxic \\
\hline
\end{tabular}

Table 7 shows the results of experiments on EOs (5\%) and extracts, against Adenovirus at concentrations of $10^{9} \mathrm{PFU} / \mathrm{mL}$ to $10^{4} \mathrm{PFU} / \mathrm{mL}$.

Table 7. Effects of essential oils and extracts on Adenovirus.

\begin{tabular}{|c|c|c|c|c|c|c|}
\hline Essential Oil 5\% & $\begin{array}{l}\text { AdV10 } \\
\text { PFU/mL }\end{array}$ & $\begin{array}{l}\text { AdV 10 } \\
\text { PFU/mL }\end{array}$ & $\begin{array}{l}\text { AdV10 } \\
\text { PFU/mL }\end{array}$ & $\begin{array}{l}\text { AdV10 } \\
\text { PFU/mL }\end{array}$ & $\begin{array}{l}\text { AdV10 } \\
\text { PFU/mL }\end{array}$ & $\begin{array}{c}\text { Adv10 } \\
\text { PFU/mL }\end{array}$ \\
\hline $\begin{array}{l}\text { Pelargonium } \\
\text { graveolens }\end{array}$ & + & + & + & + & + & + \\
\hline Rosa damascena & - & - & + & + & + & + \\
\hline \multicolumn{7}{|l|}{ Extract } \\
\hline $\begin{array}{l}\text { Pelargonium } \\
\text { graveolens } 90 \%\end{array}$ & - & + & + & + & + & + \\
\hline $\begin{array}{l}\text { Pelargonium } \\
\text { graveolens } 50 \%\end{array}$ & - & + & + & + & + & + \\
\hline $\begin{array}{l}\text { Pelargonium } \\
\text { graveolens } 25 \%\end{array}$ & - & - & + & + & + & + \\
\hline Rosa damascena $90 \%$ & + & + & + & + & + & + \\
\hline Rosa damascena $50 \%$ & + & + & + & + & + & + \\
\hline Rosa damascena $25 \%$ & + & + & + & + & + & + \\
\hline
\end{tabular}




\subsection{Life Span}

Concerning E. coli, throughout the experiment, no blue/gray colonies were detected on Tryptone Bile Glucuronic Agar (TBX agar), therefore all the samples are characterized as safe for human consumption, with regard to the E. coli microorganism. As for Salmonella spp., the experiments carried out showed that: at the weight of $25 \mathrm{~g}$ of the sample and $225 \mathrm{~mL}$ of Peptone Buffered Water, the microorganism was not detected, so the samples were considered safe for consumption, with respect to the Salmonella spp. Also, in all samples, S. aureus could not be detected. Finally, for A. niger, throughout the experiments, the samples are found to be free of mold and fungi and are considered safe for consumption.

\section{Discussion}

In the essential oil of geranium leaves, thirty-nine compounds were identified, which accounted for $67.98 \pm 5.15 \%$ of the total oil. The main components of the essential oil were citronellol $(26.7 \%)$ and geraniol (10.1\%). The other main ingredients were $\alpha$-pinene, cis-linalool oxide, cis-rose oxide, trans-rose oxide, menthone, isomenthone, $\alpha$-copaene, $\beta$-bourbonene, and citronellyl propanoate; all those have been reported previously for geranium leaves' EO [19].

A variability in the percentages of the main ingredients has been recorded. In a survey conducted in Greece and especially in the island of Crete, the two main components, citronellol and geraniol, were determined at percentages of over $25 \%$ and about $20 \%$, respectively [42]. In another study of native geranium leaf oil from Tajikistan, citronellol and geraniol were present in percentages of $37.5 \%$ and $6 \%$, respectively [19]. Accordingly, Verma et al. showed that the composition of the essential oil depends on the duration of cultivation; in that study, geraniol content was at its maximum in the crop transplanted in the month of April and citronellol content was higher in that transplanted in February, whereas all plants were harvested in June [43]. Moreover, differences of the ratio of citronellol and geraniol, according to the season of collection, have been reported; the ratio is close to one in April and June [42]. Finally, Wahab et al. [44] showed that the citronellol and the geraniol content was affected by planting location and harvest time. That work was carried out during the two successive seasons (2012/2013 and 2013/2014) to investigate the effect of different planting locations (5 different locations in Egypt) on quantity and quality of $P$. graveolens volatile oil. In most locations, the highest citronellol content was obtained in spring cut, while the lowest was obtained in autumn cut [44].

In the essential oil of rose petals, thirteen compounds were identified, representing $96.47 \pm 0.43 \%$ of the total oil (Table 1). In our study, the main components of the essential oil were identified as phenyl ethyl alcohol $(40.2 \%)$ and geraniol $(27.8 \%)$. The remaining detected compounds were less than $20 \%$. In accordance with our results, Verma et al. showed that phenyl ethyl alcohol was the main component in $R$. damascena EO of Ranisahiba cultivar (76\%), and of Noorjahan and Kannouj cultivars (80.7 and 76.7\%, respectively) at full bloom stage [45]. Similarly, in the GC analysis of EOs of $R$. damascena and R. moschata var. nastarana flowers by headspace extraction, phenyl ethyl ethanol was the dominant ingredient albeit in different amounts [46]. Interestingly, in the study of Koksal et al. [47] on damask roses from Turkey, phenyl ethyl alcohol was the major component in EO from fresh rose petals $(25.06 \%)$, and that amount significantly increased when the plant material was stored before distillation [47]. The relationship of the chemical composition with the climate has also been suggested; according to Misra et al. [48], geraniol content decreases whereas phenyl ethyl alcohol increases in colder climates and at higher altitude [48]. Accordingly, in our study, rose petals were harvested in late spring from a mountainous location $(360 \mathrm{~m}$ altitude) at a Northern Aegean island, which enjoys mild Mediterranean climate; in late spring the mean temperature does not exceed $20^{\circ} \mathrm{C}$. Therefore, differences in the rose petal EO composition can be attributed to several factors like the genotype, the cultivation, and the storage conditions.

The analysis of rose petals' extract showed eighteen identified components. The main component of the herbal extract was kaempferol-3-O-glucoside (55.4\%). The remaining 
detected components were less than $12 \%$. Compounds were mainly identified as quercetin glycosides and kaempferol derivatives by comparison of their retention time with the standards and the literature data [49-53]. As can be seen from Table 2, the kaempferol glycosides accounted for the largest percentage of the compounds that were quantified, with kaempferol-3-O-glucoside being the predominant component. This is in accordance with other studies that report that kaempferol-3-O-glucoside is the main component in rose petals of $R$. damascena [51], and in rose petals of Taif rose [53]. In this study, nine kaempferol derivatives were identified. Most of them have also been reported in other species of the Rosacae family $[49,50,52,53]$. Kaempferol deoxyhexoside and kaempferol acetyldisaccharide were previously identified in extracts of $R$. damascena from Bulgaria [51] Compounds 1 and 2 have also been determined from R. damascena from Egypt [54], and the hybrid "Jardin de Granville" from France [50], respectively. Five quercetin derivatives were identified in this study. Compounds 4 and 8 were also detected in $R$. damascena from Bulgaria [52] and from India [48]. The presence of quercetin glycosides and kaempferol aglycone has been reported also in other species of the Rosacae family, in addition to $R$. damascena, such as Rosa bourboniana Desport., Rosa brunonii Lindl., known as 'Himalayan musk rose [49], the hybrid "Jardin de Granville" [50], and Taif rose, Ward Taifi (R. damascena trigintipetala Dieck) [53].

The geranium leaves' extract analysis (Table 3) showed eleven identified components. The main components of the herbal extract were quercetin 3-O-glucoside (31.7\%), quercetin-3-O-pentosyl hexoside (28\%), and quercetin-3-O-galactoside $(24.9 \%)$. The remaining detected compounds were less than $10 \%$.

Most of the compounds were flavonol glycosides, i.e., quercetin and kaempferol derivatives. Compounds 1-9 were previously reported in a study on P. graveolens [55]. Compounds 2, 6, and 7 have been reported in other species of the Geraniacae family, except from geranium, i.e., Geranium molle L. [56] and Geranium robertianum L. [57]. Finally, to the best of our knowledge compounds 10, 11 have not been reported in Geraniacae family. Our results show that the quercetin glycosides accounted for the largest percentage of the compounds that were quantified, with quercetin-3-O-glucoside being the main component.

Our results are in line with previous studies demonstrating that rose oil and its aqueous extracts have moderate broad-spectrum antimicrobial activity $[58,59]$. Phenyl ethyl alcohol, the main rose oil constituent $(40 \%)$ in our study, has been attributed antimicrobial properties for a long time but their inhibitory actions are considered complex and generally seem to be dominated by their physicochemical properties [60]. In addition, geraniol, the other major rose oil constituent (about $28 \%$ ), and rose geranium EO constituent (10\%), and is demonstrated to have antimicrobial activity against 78 different microorganisms, such as Candida or Staphylococcus [61]. A recent study by Guimaraes et al. (2019) [62], showed that geraniol and citronellol (the major rose geranium EO constituent and a major rose oil one) are fast-acting compounds that inactivate $E$. coli and $S$. Typhimurium by inducing loss of cellular membrane integrity or function. Thus, the antimicrobial activity of rose geranium oil demonstrated is our study is explained by, and is in agreement to, previous reports $[63,64]$. Concerning their antiviral activities, limited information is available about rose geranium oil, and no information is available, as far as we know, for citronellol, geraniol, and phenyl ethyl alcohol [65].

The major component of the extract of rose petals is kaempferol-3-O-glucoside, alongside with other kaempferol glycosides, and it has been shown to have antimicrobial and antioxidant effects [66-68]. The rose geranium extract, also rich in flavonoids (quercetin glycosides), had strong antimicrobial and antiviral activity in our study; our results are in agreement with earlier reports on the antimicrobial activity of geranium extracts $[63,69]$ and quercetin derivatives [66]. The antiviral properties of quercetin and kaempferol derivatives have been reported in numerous publications [70], e.g., against influenza viruses [71,72], coronaviruses, and dengue viruses [73,74], and in particular of quercetin against adenoviruses 1 and 3 [75]; the reports indicate that those flavonols block viral entry to the host cell via specific interactions with viral attachment factors and/or membrane fusion 
proteins, suppress signaling pathways that are essential for virus gene expression, inhibit remodeling enzymes and channels (which regulate viral movement (e.g., neuraminidase)), and inhibit transcription of the viral genome and viral protein synthesis [70]. However, none of the previous studies have addressed the issue of their realistic application in foods and drinks as natural preservatives, since a final product will contain these EOs and extracts in a certain percentage, due to the organoleptic properties they confer, e.g., the strong odour.

Author Contributions: Conceptualization, P.H., F.N.L. and A.V.; methodology, P.H., F.N.L. and A.V.; validation, C.K., F.N.L. and A.V.; investigation, C.A., S.D.C., P.H.; resources, P.H., F.N.L. and A.V.; writing-original draft preparation, C.A., S.D.C., C.K.; writing-review and editing, F.N.L. and A.V.; supervision, F.N.L. and A.V.; funding acquisition, P.H., F.N.L. and A.V. All authors have read and agreed to the published version of the manuscript.

Funding: This research has been co-financed by the European Union and Greek national funds through the Regional Operational Program “Western Greece 2014-2020", under the Call "Regional research and innovation strategies for smart specialization (RIS3) in Agri-Food" in the context of the project MIS 5040309 entitled "Development of new bitters from aromatic and medicinal herbs with health benefits (Natubitters)".

Institutional Review Board Statement: Not applicable.

Informed Consent Statement: Not applicable.

Data Availability Statement: Data is contained within the article.

Conflicts of Interest: The authors declare no conflict of interest.

\section{References}

1. Swamy, M.K.; Sinniah, U.R. A comprehensive review on the phytochemical constituents and pharmacological activities of Pogostemon cablin Benth: An aromatic medicinal plant of industrial importance. Molecules 2015, 20, 8521-8547. [CrossRef]

2. Arumugam, G.; Swamy, M.K.; Sinniah, U.R. Plectranthus amboinicus (Lour.) Spreng: Botanical, phytochemical, pharmacological and nutritional significance. Molecules 2016, 21, 369. [CrossRef]

3. Sudipta, K.M.; Lokesh, P.; Rashmi, W.; Vijay, R.; Ssn, K. Phytochemical screening and in vitro antimicrobial activity of Bougainvillea spectabilis flower extracts. Int. J. Phytomedicine 2012, 4, 375-379.

4. Swamy, M.K.; Akhtar, M.S.; Sinniah, U.R. Antimicrobial Properties of Plant Essential Oils against Human Pathogens and Their Mode of Action: An Updated Review. Evid. Based Complementary Altern. Med. 2016. [CrossRef] [PubMed]

5. Oliveira, M.; Usall, J.; Vinas, I.; Anguera, M.; Gatius, F.; Abadias, M. Microbiological quality of fresh lettuce from organic and conventional production. Food Microbiol. 2010, 27, 679-684. [CrossRef] [PubMed]

6. Wetzel, K.; Lee, J.; Lee, C.S.; Binkley, M. Comparison of microbial diversity of edible flowers and basil grown with organic versus conventional methods. Can. J. Microbiol. 2010, 56, 943-951. [CrossRef] [PubMed]

7. Moore-Neibel, K.; Gerber, C.; Patel, J.; Friedman, M.; Ravishankar, S. Antimicrobial activity of lemongrass oil against Salmonella enterica on organic leafy greens. J. Appl. Microbiol. 2011, 112, 485-492. [CrossRef]

8. Pandey, A.K.; Kumar, P.; Singh, P.; Tripathi, N.N.; Bajpai, V.K. Essential Oils: Sources of Antimicrobials and Food Preservatives. Front. Microbiol. 2017, 7, 2161. [CrossRef]

9. Akhtar, B.D.; Azam, T. Antimicrobial activity of essential oils extracted from medicinal plants against the pathogenic microorganisms: A review. Biol. Sci. Pharm. Res. 2014, 2, 1-7.

10. Anupama, G.; Netravathi, D.K.; Avinash, M. Essential oils: A novel source for food preservation. J. Pharmacogn. Phytochem. 2019, 8, 2098-2101.

11. Mohanka, R.; Priyanka, A.M.J. Plant Extract as natural food preservative against spoilage Fungi from processed food. Int. J. Curr. Microbiol. Appl. Sci. 2014, 3, 91-98.

12. Kang, H.Y.; Na, S.S.; Kim, Y.K. Effects of oral care with essential oil on improvement in oral health status of hospice patients. J. Korean Acad. Nurs. 2010, 40, 473-481. [CrossRef]

13. Benelli, G.; Pavela, R.; Canale, A.; Cianfaglione, K.; Ciaschetti, G.; Conti, F.; Nicoletti, M.; Senthil-Nathan, S.; Mehlhorn, H.; Maggi, F. Acute larvicidal toxicity of five essential oils (Pinus nigra, Hyssopus officinalis, Satureja montana, Aloysia citrodora and Pelargonium graveolens) against the filariasis vector Culex quinquefasciatus: Synergistic and antagonistic effects. Parasitol. Int. 2017, 66, 166-171. [CrossRef] [PubMed]

14. Ćavar, S.; Maksimović, M. Antioxidant activity of essential oil and aqueous extract of Pelargonium graveolens L'Her. Food Control 2012, 23, 263-267. [CrossRef]

15. Akbari, M.; Kazerani, H.R.; Kamrani, A.; Mohri, M. A preliminary study on some potential toxic effects of Rosa damascena Mill. Iran. J. Vet. Res. 2013, 14, 232-236. 
16. Nunes, H.S.; Miguel, M.G. Rosa damascena essential oils: A brief review about chemical composition and biological properties. Trends Phytochem. Res. 2017, 1, 111-128.

17. Mohaddese, M. Rosa damascena as holy ancient herb with novel applications. J. Tradit. Complementary Med. 2016, 6, 10-16.

18. Viuda-Martos, M.; Mohamady, M.A.; Fernández-López, J.; Abd ElRazik, K.A.; Omer, E.A.; Pérez-Alvarez, J.A.; Sendra, E. In vitro antioxidant and antibacterial activities of essentials oils obtained from Egyptian aromatic plants. Food Control 2011, 22, 1715-1722. [CrossRef]

19. Sharopov, F.S.; Zhang, H.; Setzer, W.N. Composition of geranium (Pelargonium graveolens) essential oil from Tajikistan. Am. J. Essent. Oils Nat. Prod. 2014, 2, 13-16.

20. Van Den Dool, H.; Kratz, P.D. A generalization of the retention index system including linear temperature programmed gas liquid partition chromatography. J. Chromatogr. 1963, 11, 463-471. [CrossRef]

21. Adams, R.P. Identification of Essential Oil Components by Gas Chromatography/Mass Spectrometry, 4th ed.; Allured Publishing Corporation: Carol Stream, IL, USA, 2012.

22. National Institute of Standards and Technology. NIST WebBook. Available online: http://webbook.nist.gov/chemistry/ (accessed on 20 December 2020).

23. Mortelmans, K.; Zeiger, E. The Ames Salmonella/microsome mutagenicity assay. Fundamental and Molecular Mechanisms of Mutagenesis. Mutat. Res. 2000, 455, 29-60. [CrossRef]

24. Taherkhani, T.; Zakaria, R.A.; Taherkhani, M. Mutagenic and Anti-mutagenic properties of the essential oil of Jurinea leptoloba DC by Ames Test. Cumhuriyet University Faculty of Science. Sci. J. (CSJ) 2015, 36, 1682-1687.

25. Hughes, T.J.; Simmons, D.M.; Monteith, L.G.; Claxton, L.D. Vaporization technique to measure mutagenic activity of volatile organic chemicals in the Ames/Salmonella assay. Environ. Mutagenesis 1987, 9, 421-441. [CrossRef]

26. Isono, K.; Yourno, J. Chemical carcinogens as frameshift mutagens: Salmonella DNA sequence sensitive to mutagenesis by polycyclic carcinogens. Proc. Natl. Acad. Sci. USA 1974, 71, 1612-1617. [CrossRef] [PubMed]

27. Barnes, W. Base-sequence analysis of His+ revertants of the hiG46 missense mutation in Salmonella typhimurium. Environ. Mutagenesis 1982, 4, 297.

28. Saderi, H.; Abbasi, M. Evaluation of anti-adenovirus activity of some plants from Lamiaceae family grown in Iran in cell culture. Afr. J. Biotechnol. 2011, 10, 17546-17550.

29. Araki, A.; Noguchi, T.; Kato, F.; Matsushima, T. Improved method for mutagenicity testing of gaseous compounds by using a gas sampling bag. Mutat. Res. 1994, 307, 335-344. [CrossRef]

30. Simmon, V.F.; Kauhanen, K.; Tardiff, R.G. Mutagenic activities of chemicals identified in drinking water. In Progress in Genetic Toxicology; Scott, D., Bridges, B.A., Sobels, F.H., Eds.; Elsevier/North-Holland: Amsterdam, The Netherlands, 1977; pp. 249-258.

31. Zeiger, E.; Anderson, B.; Haworth, S.; Lawlor, T.; Mortelmans, K. Salmonella mutagenicity tests: V. Results from the testing of 311 chemicals. Environ. Mol. Mutagenesis 1992, 19 (Suppl. 21), 1-141. [CrossRef]

32. Hengstler, J.G.; Oesch, F. Ames Test. In Encyclopedia of Genetics; Brenner, S., Miller, J., Eds.; Academic Press: New York, NY, USA, 2001; pp. 51-54.

33. Oliveira, N.D.M.S.; Resende, M.R.; Morales, D.A.; de Ragão Umbuzeiro, G.; Boriollo, M.F.G. In vitro mutagenicity assay (Ames test) and phytochemical characterization of seeds oil of Helianthus annuus Linné (sunflower). Toxicol. Rep. 2016, 3, 733-739. [CrossRef]

34. Vaughan, D.J.; Baptista, J.A.; Perdomo, G.R.; Krepinsky, J.J. The involvement of dimethyl sulfoxide in a bacteriotoxic response of the Ames assay tester strains TA98 and TA100. Mutat. Res. 1989, 226, 39-42. [CrossRef]

35. Erdemgil, F.Z.; Ilhan, S.; Korkmaz, F.; Kaplan, C.; Mercangöz, A.; Arfan, M.; Ahmad, S. Chemical Composition and Biological Activity of the Essential Oil of Perovskia atriplicifolia From Pakistan. Pharm. Biol. 2007, 45, 324-331. [CrossRef]

36. Maron, D.M.; Ames, B.N. Revised method for the Salmonella mutagenicity test. Mutat. Res. 1983, 113, 173-215. [CrossRef]

37. Mudyiwa, M.; Muredzi, P.; Nyati, H. Isolation of Citric acid Producing Asp. Niger Strains; LAP Lambert Academic Publishing: Chisinau, Republic of Moldova, 2013.

38. Toscano-Garibay, J.D.; Arriaga-Alba, M.; Sánchez-Navarrete, J.; Mendoza-García, M.; Flores-Estrada, J.J.; Moreno-Eutimio, M.A.; Espinosa-Aguirre, J.J.; González-Ávila, M.; Ruiz-Pérez, N.J. Antimutagenic and antioxidant activity of the essential oils of Citrus sinensis and Citrus latifolia. Sci. Rep. 2017, 7, 11479. [CrossRef] [PubMed]

39. Humphries, R.M.; Abbott, A.N.; Hindler, J.A. Understanding and addressing CLSI breakpoint revisions: A primer for clinical laboratories. J. Clin. Microbiol. 2019, 57, e00203-19. [CrossRef] [PubMed]

40. Barel, A.O.; Paye, M.; Maibach, H.I. Handbook of Cosmetic Science and Technology, 2nd ed.; CRC Press: Boca Raton, FL, USA, 2005; Chapter 64, Test Organisms.

41. Rijal, N. Potato Dextrose Agar (PDA): Principle, composition and colony characteristics. Med. Microbiol. Guide Cult. Media Used Microbiol. 2015. Available online: https://microbeonline.com/potato-dextrose-agar-pda-principle-composition-colonycharacteristics / (accessed on 15 April 2021).

42. Jeddou, K.B.; Benchennouf, A.; Jancheva, M.; Grigorakis, S.; Lydakis-Simantiris, N. Seasonal variation effects on essential oil contend and composition of Pelargonium graveolens cultivated in Greece, Crete. In Proceedings of the International Conference "Climate Changing Agriculture", Chania, Greece, 29 August-2 September 2017; pp. 146-150. 
43. Verma, R.S.; Verma, R.K.; Yadav, A.K.; Chauhan, A. Changes in the essential oil composition of rose-scented geranium (Pelargonium graveolens L'Herit. ex Ait.) due to date of transplanting under hill conditions of Uttarakhand. Indian J. Nat. Prod. Resour. 2010, $1,367-370$.

44. Abd El-Wahab, A.; Toaima, M.; Hamed, S. Effect of different planting locations in Egypt on volatile oil of geranium (Pelargonium graveolens L.) plant. J. Basic Appl. Res. 2016, 2, 522-533.

45. Verma, R.S.; Padalia, R.C.; Chauhan, A.; Singh, A.; Yadav, A.K. Volatile constituents of essential oil and rosewater of damask rose (Rosa damascena Mill.) cultivars from North Indian hills. Nat. Prod. Res. 2011, 25, 1577-1584. [CrossRef]

46. Karami, A.; Jandoust, S. Comparison Scent Compound Emitted from Flowers of Damask Rose and Persian Musk Rose. Med. Aromat. Plants 2016, 5, 4. [CrossRef]

47. Koksall, N.; Aslancan, H.; Sadighazadi, S.; Kafkas, E. Chemical investigation on Rose damascena Mill. volatiles; Effect of storage and drying condition. Acta Sci. Pol. Hortorum Cultus 2015, 14, 105-114.

48. Misra, A.; Sharma, S.; Singh, A.; Patra, N.K. Influence of topographical and edaphic factors on rose. Flower. Qual. Quant. 2002, $33,2771-2780$

49. Kumar, N.; Bhandari, P.; Singh, B.; Bari, S.S. Antioxidant activity and ultra-performance LC-electrospray ionization-quadrupole time-of-flight mass spectrometry for phenolics-based fingerprinting of Rose species: Rosa damascena, Rosa bourboniana and Rosa brunonii. Food Chem. Toxicol. 2009, 47, 361-367. [CrossRef] [PubMed]

50. Riffault, L.; Destandau, E.; Pasquier, L.; André, P.; Elfakir, C. Phytochemical analysis of Rosa hybrida cv. 'Jardin de Granville' by HPTLC, HPLC-DAD and HPLC-ESI-HRMS: Polyphenolic fingerprints of six plant organs. Phytochemistry 2014, 99, 127-134. [CrossRef] [PubMed]

51. Schieber, A.; Mihalev, K.; Berardini, N.; Mollov, P.; Carle, R. Flavonol Glycosides from Distilled Petals of Rosa damascena Mill. Z. Für Nat. C 2005, 60, 379-384. [CrossRef] [PubMed]

52. Cai, Y.Z.; Xing, J.; Sun, M.; Zhan, Z.Q.; Corke, H. Phenolic Antioxidants (Hydrolyzable Tannins, Flavonols, and Anthocyanins) Identified by LC-ESI-MS and MALDI-QIT-TOF MS from Rosa chinensis Flowers. J. Agric. Food Chem. 2005, 53, 9940-9948. [CrossRef] [PubMed]

53. Abdel-Hameed, E.S.S.; Bazaid, S.A.; Salman, M.S. Characterization of the Phytochemical Constituents of Taif Rose and Its Antioxidant and Anticancer Activities. BioMed Res. Int. 2013, 2013, 13. [CrossRef]

54. Mohsen, E.; Younis, I.Y.; Farag, M.A. Metabolites profiling of Egyptian Rosa damascena Mill. flowers as analyzed via ultra-highperformance liquid chromatography-mass spectrometry and solid-phase microextraction gas chromatography-mass spectrometry in relation to its anti-collagenase skin effect. Ind. Crop. Prod. 2020, 155, 112818.

55. Al-Sayed, E.; Martiskainen, O.; el-Din, S.H.S.; Sabra, A.N.A.; Hammam, O.A.; El-Lakkany, N.M. Protective effect of Pelargonium graveolens against carbon tetrachloride-induced hepatotoxicity in mice and characterization of its bioactive constituents by HPLC-PDA-ESI-MS/MS analysis. Med. Chem. Res. 2015, 24, 1438-1448. [CrossRef]

56. Graça, V.C.; Dias, M.I.; Barros, L.; Calhelha, R.C.; Santos, P.F.; Ferreira, I.C. Fractionation of the more active extracts of Geranium molle L.: A relationship between their phenolic profile and biological activity. Food Funct. 2018, 9, 2032. [CrossRef]

57. Graça, V.C.; Barros, L.; Calhelha, R.C.; Dias, M.I.; Ferreira, I.C.; Santos, P.F. Bio-guided fractionation of extracts of Geranium robertianum L.: Relationship between phenolic profile and biological activity. Ind. Crop. Prod. 2017, 108, 543-552. [CrossRef]

58. Ulusoy, S.; Boşgelmez-Tınaz, G.; Seçilmiş-Canbay, H. Tocopherol, Carotene, Phenolic Contents and Antibacterial Properties of Rose Essential Oil, Hydrosol and Absolute. Curr. Microbiol. 2009, 59, 554-558. [CrossRef] [PubMed]

59. Shohayeb, M.; Abdel-Hameed, E.S.S.; Bazaid, S.A.; Maghrabi, I. Antibacterial and Antifungal Activity of Rosa damascena MILL. Essential Oil, Different Extracts of Rose Petals. Glob. J. Pharmacol. 2014, 8, 1-7.

60. Etschmann, M.; Bluemke, W.; Sell, D.; Schrader, J. Biotechnological production of 2-phenylethanol. Appl. Microbiol. Biotechnol 2002, 59, 1-8.

61. Lira, M.H.P.D.; Andrade Júnior, F.P.D.; Moraes, G.F.Q.; Macena, G.D.S.; Pereira, F.D.O.; Lima, I.O. Antimicrobial activity of geraniol: An integrative review. J. Essent. Oil Res. 2020, 32, 187-197. [CrossRef]

62. Guimarães, A.C.; Meireles, L.M.; Lemos, M.F.; Guimarães, M.C.C.; Endringer, D.C.; Fronza, M.; Scherer, R. Antibacterial Activity of Terpenes and Terpenoids Present in Essential Oils. Molecules 2019, 24, 2471. [CrossRef]

63. Hsouna, A.B.; Hamdi, N. Phytochemical composition and antimicrobial activities of the essential oils and organic extracts from Pelargonium graveolens growing in Tunisia. Lipids Health Dis. 2012, 11, 167. [CrossRef]

64. Džamić, A.M.; Soković, M.D.; Ristić, M.S.; Grujić, S.M.; Mileski, K.S.; Marin, P.D. Chemical composition, antifungal and antioxidant activity of Pelargonium graveolens essential oil. J. Appl. Pharm. Sci. 2014, 4, 1-5.

65. Ma, L.; Yao, L. Antiviral Effects of Plant-Derived Essential Oils and Their Components: An Updated Review. Molecules 2020, 25, 2627. [CrossRef]

66. Farhadi, F.; Khameneh, B.; Iranshahi, M.; Iranshahy, M. Antibacterial activity of flavonoids and their structure-activity relationship: An update review. Phytother. Res. 2019, 33, 13-40. [CrossRef]

67. Taiwo, F.O.; Oyedeji, O.; Osundahunsi, M.T. Antimicrobial and Antioxidant Properties of kaempferol-3-O-glucoside and 1-(4Hydroxyphenyl)-3-phenylpropan-1-one Isolated from the Leaves of Annona muricata (Linn.). J. Pharm. Res. Int. 2019, $26,1-13$. [CrossRef]

68. Achika, J.I.; Ayo, R.G.; Oyewale, A.O.; Habila, J.D. Flavonoids with antibacterial and antioxidant potentials from the stem bark of Uapaca heudelotti. Heliyon 2020, 6, e03381. [CrossRef] 
69. Ali, I.B.E.; Tajini, F.; Boulila, A.; Jebri, M.A.; Boussaid, M.; Messaoud, C.; Sebaï, H. Bioactive compounds from Tunisian Pelargonium graveolens (L'Hér.) essential oils and extracts: $\alpha$-amylase and acetylcholinesterase inhibitory and antioxidant, antibacterial and phytotoxic activities. Ind. Crop. Prod. 2020, 158, 112951. [CrossRef]

70. Zakaryan, H.; Arabyan, E.; Oo, A.; Zandi, K. Flavonoids: Promising natural compounds against viral infections. Arch. Virol. 2017, 162, 2539-2551. [CrossRef]

71. Gansukh, E.; Nile, A.; Kim, D.H.; Oh, J.W.; Nile, S.H. New insights into antiviral and cytotoxic potential of quercetin and its derivatives-A biochemical perspective. Food Chem. 2021, 1, 127508. [CrossRef] [PubMed]

72. Kai, H.; Obuchi, M.; Yoshida, H.; Watanabe, W.; Tsutsumi, S.; Park, Y.K.; Matsuno, K.; Yasukawa, K.; Kurokawa, M. In vitro and in vivo anti-influenza virus activities of flavonoids and related compounds as components of Brazilian propolis (AF-08). J. Funct. Foods 2014, 8, 214-223. [CrossRef]

73. Chiow, K.H.; Phoon, M.C.; Putti, T.; Tan, B.K.; Chow, V.T. Evaluation of antiviral activities of Houttuynia cordata Thunb. extract, quercetin, quercetrin and cinanserin on murine coronavirus and dengue virus infection. Asian Pac. J. Trop. Med. 2016, 9, 1-7. [CrossRef]

74. Solnier, J.; Fladerer, J.P. Flavonoids: A complementary approach to conventional therapy of COVID-19? Phytochem. Rev. 2020. [CrossRef] [PubMed]

75. Chen, C.H.; Chou, T.W.; Cheng, L.H.; Ho, C.W. In vitro anti-adenoviral activity of five Allium plants. J. Taiwan Inst. Chem. Eng. 2011, 42, 228-232. [CrossRef] 